\title{
Genome-wide identification and expression analyses of R2R3- MYB transcription factor genes from two Orchid species
}

\author{
Honghong Fan Equal first author, 1 , Manli Cui ${ }^{\text {Equal first author, } 1}{ }^{,}$, Ninghong $\mathrm{Li}^{1}{ }^{1}$, Xujuan $\mathrm{Li}^{1}{ }^{1}$, Yuxuan Liang ${ }^{2}$, Lin Liu ${ }^{1}$, Yongping Cai \\ Corresp., 1 , Yi Lin Corresp. 1 \\ ${ }^{1}$ School of Life Sciences, Anhui Agricultural University, Hefei, China \\ ${ }^{2}$ Faculty of Forestry, University of British Columbia, Vancouver, Canada \\ Corresponding Authors: Yongping Cai, Yi Lin \\ Email address: swkx12@ahau.edu.cn, linyi1957@126.com
}

MYB transcription factors play important roles in different plant biological processes during plant growth, development and stress response. In this study, 101 (DoMYB1-101) and 99 (PaMYB1-99) R2R3-MYB genes were identified in the genomes of Dendrobium officinale and Phalaenopsis aphrodite, respectively. To classify the isolated candidate genes, the R2R3-MYB genes from $A$. thaliana were selected as references. As a result, all identified $D O M Y B$ and PaMYB genes were classified into 22 subfamilies. Phylogenetic analysis revealed that S21 had the largest number of members of all the subfamilies. The numbers of introns, exons and conserved sequences in all of the identified genes are different. In addition, 20 DoMYB genes from six subfamilies were selected for further analysis of tissuespecific expression and responses to various abiotic stresses treatments. The results showed that all of the DoMYB genes in S4 and S19 subfamily exhibited the highest relative expression levels in flowers. And five DoMYB genes including DoMYB31, DoMYB40, DoMYB49, DoMYB52 and DoMYB54, responded to the stress response. These results may provide useful information for further studies of the R2R3-MYB gene family. 


\section{Genome-wide identification and expression analyses of R2R3-MYB 2 transcription factor genes from two Orchid species}

3 Honghong Fan ${ }^{\dagger 1}$, Manli Cui ${ }^{\dagger 1}$, Ninghong $\mathrm{Li}^{1}$, Xujuan $\mathrm{Li}^{1}$, Yuxuan Liang ${ }^{2}$, Lin Liu ${ }^{1}$, Yongping $4 \mathrm{Cai}^{* 1}$, Yi Lin*1

5 1. School of Life Sciences, Anhui Agricultural University, Hefei 230036, China;

6 2. Faculty of Forestry, University of British Columbia, Vancouver V6T1Z4, Canada;

7 hhfan0551@126.com (H.F.);1289121655@qq.com (M.C.); lnh0411@163.com(N.L.)

8 1xj152567@163.com (X.L.); liulin2018@ahau.edu.cn (L.L.).

9 *Correspondence:swkx12@ahau.edu.cn (Yongping Cai); linyi1957@126.com (Yi Lin)

10 Tel: +86-551-65786137 (Yongping Cai); +86-551-65786340 (Yi Lin)

$11 \dagger$ These authors contributed equally to this work. 


\section{Abstract}

14 MYB transcription factors play important roles in different plant biological processes during 15 plant growth, development and stress response. In this study, 101 (DoMYB1-101) and 99 16 (PaMYB1-99) R2R3-MYB genes were identified in the genomes of Dendrobium officinale and 17 Phalaenopsis aphrodite, respectively. To classify the isolated candidate genes, the R2R3-MYB 18 genes from $A$. thaliana were selected as references. As a result, all identified DoMYB and 19 PaMYB genes were classified into 22 subfamilies. Phylogenetic analysis revealed that S21 had 20 the largest number of members of all the subfamilies. The numbers of introns, exons and 21 conserved sequences in all of the identified genes are different. In addition, 20 DoMYB genes 22 from six subfamilies were selected for further analysis of tissue-specific expression and 23 responses to various abiotic stresses treatments. The results showed that all of the DoMYB genes 24 in S4 and S19 subfamily exhibited the highest relative expression levels in flowers. And five 25 DoMYB genes including DoMYB31, DoMYB40, DoMYB49, DoMYB52 and DoMYB54, 26 responded to the stress response. These results may provide useful information for further studies 27 of the R2R3-MYB gene family.

28 Key words: Dendrobium officinale; Phalaenopsis aphrodite; Orchidaceae; R2R3-MYB; Gene 29 family 


\section{Introduction}

As a superfamily of transcription factors (TFs), MYB proteins comprise one of largest gene families in plants. They play important roles in controlling metabolism regulation and abiotic or biotic responses during the whole processes of plant growth and development (Chen et al., 2005; Du et al., 2012; Dubos et al., 2010; Peng et al., Nesi et al., 2001). For example, MYB TFs are involved in the regulation of various phytochemical biosynthesis pathways (Adato et al., 2009). They are closely related to the regulation of several developmental processes, secondary cell wall biosynthesis, the development of stem apical meristems, and the synthesis of plant lignin (Zhong et al., 2008; Kirik et al., 1998).

The MYB gene was firstly identified in maize and then successively isolated from many other plants. MYB TFs contained a highly conserved DNA binding domain (R) with 50 to 53 amino acids and a conserved tryptophan at every 18 amino acids. Based on the number of special $\mathrm{R}$ domains, MYB TFs can be divided into four categories, namely, R1R2R3-MYB, R2R3-MYB, R1-MYB, and 4R-MYB (Dubos et al., 2010). Among them, R2R3-MYB contains two conserved domains $(2 \mathrm{R})$ with five tryptophan residues in the repeat sequence, which forms a helix-turnhelix motif at the N-terminus and plays a vital role in maintaining the stability of the MYB TFs (Rabinowicz et al., 1999; Stracke et al., 2001).

Recently, the $R 2 R 3-M Y B$ gene family has been identified in many plants. There are at least 126, 109, 157 and 192 members of R2R3-MYB genes in Arabidopsis thaliana, Oryza sativa, Zea mays and Populus trichocarpa, respectively (Dubos et al., 2010; Du et al., 2012; Katiyar et al., 2012; Wilkins et al., 2009). Clustering analysis of the $R 2 R 3-M Y B$ gene family in $A$. thaliana revealed that the R2R3-MYB genes could be divided into 25 subfamilies (Matus et al., 2009; Stracke et al., 2001). Of all these subfamilies, the S4 subfamily was involved in the synthesis of various alkenes in Oil palm ( $\mathrm{Li}$ et al., 2017). The S6 subfamily was proved to be closely related to the synthesis of carotenoids and anthocyanins ( $\mathrm{Li}$ et al., 2017). The $\mathrm{S} 7$ subfamily was demonstrated to play a regulatory role in the terpenoid synthesis pathway in A. thaliana and $M$. spicata (Reddy et al., 2017; Stracke et al., 2007).

The orchid species are widely distributed in the world. For their ornamental value and medical use, four species of Orchidaceae including Apostasia shenzhenica, Vanilla fragrans, Phalaenopsis aphrodite and Dendrobium officinale have completed the whole genome sequencing (Zhang et al., 2017). Further biological evolution analysis revealed that orthologous genes might contribute to evolutionary innovations of nonfunctional, subfunctional or new 
63 functional genes after the evolutions of common ancestors for plants (Riechmann et al., 2000).

64 MYB TFs are involved in all aspects of plant development and metabolism. Nevertheless, the

65 reports on MYB TFs in orchids are few. In this study, the $R 2 R 3-M Y B$ gene families were 66 identified from D. officinale $(D o M Y B)$ and P. aphrodite (PaMYB). The analysis of the 67 classification, phylogenetics, and chromosomal distribution was conducted. Otherwise, the 68 expression patterns of the $D o M Y B$ genes were analyzed. The results may provide novel insights 69 into the roles of MYB transcription factors in D. officinale, P. aphrodite, and other orchid plants.

\section{Materials \& Methods}

Identification and classification of $R 2 R 3-M Y B$ genes in $D$. officinale and $P$. aphrodite

The genomes of D. officinale (http://herbalplant.ynau.edu.cn/) and P.aphrodite 73 (http://orchidstra2.abrc.sinica.edu.tw/orchidstra2/padownload.php) were downloaded to identify the candidate $R 2 R 3-M Y B$ genes as previously described by Cao et al. (2016). Specifically, the Hidden Markov Model (HMM) in Pfam2 database was used to generate conserved domains (PF00249). It was then used to blast against the genome (E-value $<1 \mathrm{e}^{-3}$ ) to obtain candidate R2R3-MYB genes. All candidate sequences were confirmed with the SMART tool (http://smart.embl-heidelberg.de). The uncertain $R 2 R 3-M Y B$ proteins were filtered out. For further analysis of the phylogenetic relationships for the $R 2 R 3-M Y B$ gene members in $D$. officinale and $P$. aphrodite, a phylogenetic dendrogram was constructed based on the full sequences of candidate proteins using MEGA5.2 software with the neighbor-joining (NJ) method. In detail, a phylogeny test was performed using the bootstrap method with 1000 replicates, substitution with the Possion model, and Gaps/Missing Data treatment with pairwise deletion. The uniform rates were used as the rates among sites. The number of threads is 7 (Tamura et al., 2011). The gap open and gap extend are -2.9 and 0 , respectively. According to the classification of MYB subfamily in $A$. thaliana, the $R 2 R 3-M Y B$ gene members were eventually divided into 25 subfamilies (Matus et al., 2009; Stracke et al., 2001).

Intron-exon structure and domain analysis of R2R3-MYB genes from $D$. officinale and $P$. aphrodite

The Gene Structure Display Server 2.04 (GSDS) was used to analyze the exons-introns structures of the obtained full sequences (Cao et al., 2016). The MEME suite (Multiple Expectation Maximization for Motif Elicitation, version 5.1.0) was employed to analyze the conserved motif (Bailey et al., 2006). The parameters were set as follows, 0 or 1 occurrence per 
94 sequence to be distributed in sequences; maximum number of motifs to find, 8; minimum width

95 of motif, 6; maximum width of motif, 100; and the motif must be present in all members within

96 the same subgroup. Subsequently, MAST XML output was used to analysis protein databases

97 and finally redraw motif pattern. The annotations of the conservative patterns were performed

98 using Pfam and SMART.

99 Calculations of synonymous substitutions (Ks) and nonsynonymous substitutions (Ka)

100 According to the phylogenetic tree, two sequences of candidate genes with similar genetic 101 relationships were isolated. The gene pairs were compared using DNAMAN software. Gene 102 pairs with a consistency greater than 60\% were selected for the calculation of Ka (non103 synonymous substitution) and Ks (synonymous substitution). The values of Ka/Ks, Ka, and Ks 104 were calculated using DnaSP 5.0 software (Librado et al., 2009). A Ka/Ks ratio of 1.0 has been 105 suggested to be a useful cut-off value to identify genes under positive selection. Generally, $106 \mathrm{Ka} / \mathrm{Ks}_{\mathrm{s}}<1.0$ indicates purifying or negative selection. $\mathrm{Ka} / \mathrm{Ks}=1.0$ shows neutral selection, and $107 \mathrm{Ka} / \mathrm{Ks}>1.0$ means positive selection (Zhang et al., 2006).

108 R domain and gene ontology (GO) annotation analysis

109 The sequences of the R2 and R3 domains of 101 DoMYBs and 99 PaMYBs were aligned using 110 MEGA 5.0 to identify their features in the sequences. The multiple alignment files for these 111 domains were submitted to WebLogo (http://weblogo.berkeley.edu/logo.cgi) using the default 112 settings to acquire sequence logos as previously described by Cao et al. (2016). The protein 113 sequences of DoMYBs and PaMYBs were aligned to the NCBI non-redundant protein database

114 by BLASTp using Blast2GO software with the default parameters (Conesa et al., 2005). The

115 functions of the R2R3-MYB proteins were predicted by GO annotation analysis. The GO 116 classifications were performed using the WEGO online tool (Ye, 2006).

\section{Physical distribution of $\mathrm{PaMYB}$ genes in chromosomes}

118 To understand the chromosomal location of PaMYBs, the GFF (General Feature Format) file 119 containing the $P$. aphrodite chromosome information was downloaded from the website 120 (http://orchidstra2.abrc.sinica.edu.tw/orchidstra2/pagenome.php) (Chao et al., 2018). MapInspect 121 software was used to visualize physical location information. DNAMAN was used to compare 122 the similarity of the $\operatorname{PaR} 2 R 3-M Y B$ cluster.

123 Bioinformatics analysis of $R 2 R 3-M Y B$ genes expression in $D$. officinale

124 The transcriptome of $D$. officinale were downloaded from the NCBI SRA database with the 125 accession number PRJNA348403. Clean reads were obtained by removing the low-quality base 
126 calls $(\mathrm{Q}<20)$ using FASTX-toolkit (http://hannonlab.cshl.edu/fastx_toolkit), then mapped to the

127 reference genome using TopHat2 software with the default parameters and assembled using

128 Cufflinks software (Kim et al., 2013; Trapnell et al., 2012). The expression profiles were

129 presented using MeV 4.9 with Pearson correlation using average linkage clustering.

130 To verify the transcriptome data, 20 genes were selected to perform qRT-PCR analysis. Three

131 biological repeats were conducted. The average data was used to construct heatmap by MeV 4.9

132 with Pearson correlation using average linkage clustering.

133 A co-expression network of 20 DoMYB genes in response to five different treatments was 134 constructed based on the Pearson correlation coefficients (PCCs). It was better to understand the 135 topological relationships between stress-responsive DoMYB genes. All of the available gene 136 expression data were averaged, and the PCC was calculated between any pair of 20 genes (Tang 137 et al., 2013). All 20 genes were selected to evaluate a co-expression network based on the PCCs 138 at a 0.05 significance level (p-value) using Cytoscape 3.3 (http://www.cytoscape.org).

\section{Expression analysis $R \mathbf{R} R \mathbf{R}-\mathbf{M Y B}$ genes under different treatments in $D$. officinale}

140 One-year-old plantlets of $D$. officinale were cultivated in an incubator at $25^{\circ} \mathrm{C}$ with $12 \mathrm{~h}$ light 141 and $12 \mathrm{~h}$ dark. The protocorms of $D$. officinale were cultivated at $25^{\circ} \mathrm{C}$ in the dark. For the stress 142 treatment, an equal mass protocorm of the same period was placed in MS liquid medium with an 143 additional $300 \mathrm{mM} \mathrm{NaCl}, 300 \mathrm{mM}$ mannitol and $100 \mu \mathrm{M}$ abscisic acid (ABA), respectively.

144 Then, protocorms with the same quality were collected at $0,1,2,3,4$, and $7 \mathrm{~d}$ for further 145 experiment. Moreover, for the treatment with hormone, the protocorms with the same size in the 146 same period were cultivated in MS liquid medium additionally containing $100 \mu \mathrm{M}$ methyl 147 jasmonate (MeJA) and $100 \mu \mathrm{M}$ salicylic acid (SA), respectively. The protocorms were collected 148 at $0,2,4,8,24,48$ and $72 \mathrm{~h}$. Protocorms, $0 \mathrm{~h}$ cultivated in a different medium, were selected as 149 controls, respectively. Each experiment was repeated three times.

150 Total RNA was extracted by using RNAprep Pure Plant Kit (Tiangen, China). Primer Premier 1515.0 was used to design the corresponding primers of DoMYB (Supplementary Table S4). The 152 gene $\beta$-actin was selected as an internal reference (GeneBank ID: JX294908). The qPCR system 153 consisted of $12.5 \mu \mathrm{l}$ of SYBR ${ }^{\circledR}$ Premix Ex TaqTM II, $2 \mu \mathrm{l}$ of cDNA, $2 \mu \mathrm{l}$ primers and $8.5 \mu \mathrm{l}$ of $154 \mathrm{ddH}_{2} \mathrm{O}$. The PCR reaction procedure was as follows: $95^{\circ} \mathrm{C}$ for $30 \mathrm{~s}, 95^{\circ} \mathrm{C}$ for $5 \mathrm{~s}, 60{ }^{\circ} \mathrm{C}$ for $30 \mathrm{~s}$, 15540 cycles (Jin et al., 2013). The relative expression level of genes was calculated using the $2^{-\Delta \Delta \mathrm{Ct}}$ 156 method (Livak et al., 2001). Three biological repeats were conducted. 


\section{Results}

158 Phylogenetic analysis and classification of the $R 2 R 3-M Y B$ genes from $D$. officinale and $P$.

159

160

161

162

163

164

165

166

167

168

169

170

171

172

173

174

175

176

177

178

179

180

181

182

183

184

185

186

187

\section{aphrodite}

As was shown in Fig. 1, total of 101 DoMYBs and 99 PaMYBs were identified (Supplementary Table S1). Then, 126 R2R3-MYB genes from Arabidopsis (AtMYBs) were selected as references to construct the neighbor-joining tree. The results showed that all identified $R 2 R 3-M Y B$ genes could be divided into 22 subgroups with the number of members ranging from 1 to 16 . Of 22 subgroups, the subfamily S21 contained the most members of R2R3-MYB genes with 16 DoMYBs and 11 PaMYBs, followed by S14 subfamily with 10 DoMYBs and 11 PaMYBs. The subfamily S18 contained 12 DoMYBs and 6 PaMYBs. Interestingly, nine subfamilies, including $\mathrm{S} 1, \mathrm{~S} 3, \mathrm{~S} 4, \mathrm{~S} 9, \mathrm{~S} 13, \mathrm{~S} 16, \mathrm{~S} 17, \mathrm{~S} 19$, and $\mathrm{S} 23$, contained the same numbers of R2R3-MYBs from both $D$. officinale and P. aphrodite. For example, both six members of DoMYBs and PaMYBs were divided into S17 subfamily. S1 and S4 subfamilies included both five R2R3-MYBs for $D$. officinale and $P$. aphrodite. The subfamily S13 contained four R2R3-MYBs from both $D$. officinale and P. aphrodite. Otherwise, no R2R3-MYB genes were classified into the subfamilies $\mathrm{S} 6, \mathrm{~S} 12$, and $\mathrm{S} 15$ from the two Orchidaceae species. So, the distributions of most $R 2 R 3-M Y B$ genes were similar between $D$. officinale and P. aphrodite.

\section{Gene structure analysis of the R2R3-MYB protein from $D$. officinale and $P$. aphrodite}

The R2R3-MYB protein sequences of $D$. officinale and $P$. aphrodite were used to create a phylogenetic tree using MEGA5.2 software (Fig. 2A and Supplementary Fig. S1A). The conserved motifs were identified using the MEME tool. The results showed that most of R2R3MYB proteins in the same subfamily contained similar motifs, which futher verifying the closeness of their evolutionary relationship with the phylogenetic tree (Fig. 2B and Supplementary Fig. S1B). Nevertheless, motif 3, which didn't belong to the conserved domain $\mathrm{R} 2$ or R3, was included in almost half of R2R3-MYB members in D. officinale and P. aphrodite. Otherwise, the subfamilies $\mathrm{S} 4, \mathrm{~S} 8, \mathrm{~S} 9, \mathrm{~S} 10, \mathrm{~S} 11$ and $\mathrm{S} 13$, all contained the motif 4 in $D$. officinale. Furthermore, the gene structure analysis of the R2R3-MYB genes revealed that most of these genes consisted of three exons and two introns (Fig. 2C and Supplementary Fig. S1C). These results indicated that the intron patterns were highly conserved.

Strong purifying selection analysis of the R2R3-MYB genes from $D$. officinale and $P$. aphrodite 
188 To examine the selection pressure on the $R 2 R 3-M Y B$ genes, 16 gene pairs between $P$. 189 aphrodite and $D$. officinale, 19 gene pairs of $P$. aphrodite and four gene pairs of $D$. officinale 190 with close genetic affinities were selected to calculate the $\mathrm{Ka} / \mathrm{Ks}$ ratio. As a result, of 16 gene 191 pairs between two species, $87 \%$ showed a rate less than 0.3 . Of the 19 gene pairs from $D$. 192 officinale, $79 \%$ exhibited a $\mathrm{Ka} / \mathrm{Ks}$ ratio between 0 and 0.5 , and only four gene pairs scored a $193 \mathrm{Ka} / \mathrm{Ks}$ ratio greater than 0.5 . Moreover, the $\mathrm{Ka} / \mathrm{Ks}$ ratios of the four gene pairs from $P$. aphrodite 194 were between 0 and 1 (Supplementary Table S2). These results implied that most of the R2R3195 MYB gene pairs from $D$. officinale and $P$. aphrodite mainly evolved under the influence of 196 purifying selection.

197 Gene ontology annotation and $\mathbf{R}$ domain characteristics analysis the $R 2 R 3-M Y B$ genes 198 from $D$. officinale and $P$. aphrodite

199 The functions of the R2R3-MYB proteins were predicted by GO annotation analysis. Based on 200 amino acid similarity, the R2R3-MYB proteins from two Orchidaceae species were classified 201 into three categories: biological process (BP), cellular component (CC), and molecular function 202 (MF) (Supplementary Table S5, Fig. S2). The majority of the R2R3-MYB proteins from $D$. 203 officinale and $P$. aphrodite were enriched in two categories: biological process and molecular 204 function. Only a small portion of the R2R3-MYB proteins was enriched in the cellular 205 component (Supplementary Fig. S2AC).

206 To analyze the presence of conserved sequences at particular positions, WebLogo was used to 207 generate sequence logos. As is shown in Fig. 3, A and B indicated the repeats of the R2 and R3 208 sequences of D. officinale, respectively. C and D showed the repeats of the R2 and R3 sequences 209 of $P$. aphrodite, respectively. These results indicated that most amino acids are conservatively 210 presented in the R2 and R3 repeats between two Orchidaceae species (Fig. 3). Otherwise, some 211 amino acids are more conservative. For example, tryptophan was identified at the sites 212 approximately per every 18 amino acids. There were three tryptophans in R2 and two in R3.

\section{Chromosomal location analysis of the PaMYB genes}

214 To study the relationship between genetic divergence and gene duplication within the $R 2 R 3-$ $215 M Y B$ gene family in $P$. aphrodite, the locations and distribution of the $R 2 R 3-M Y B$ genes were 216 analyzed. As a result, of the 99 PaMYB genes, 85 genes were mapped and distributed in 17 217 chromosomes of $P$. aphrodite. The distribution of genes was uneven. Chromosome 9 contained 218 the most R2R3-MYB genes (10 PaMYB genes), followed by chromosome 18, which contained 9 219 PaMYB genes. The other chromosomes contained $R 2 R 3-M Y B$ genes, ranging from 1 to 8 , 
220 respectively (Supplementary Fig. S3). In addition, 33 pairs of R2R3-MYB proteins were isolated 221 with similar sequences by using sequence alignment in DNAMAN (Supplementary Table S6).

222 Of these proteins, two pairs were proved to be segmental duplication for their high similarity 223 values (PaMYB64/PaMYB65, PaMYB73/PaMYB91). PaMYB64 and PaMYB65 have an 224 identical sequence with the same position on chromosome 9 (Supplementary Table S6). 225 PaMYB73 and PaMYB91 showed a high similarity value of 87.25\% (Supplementary Table S6).

226

227

228

229

230

231

232

233

234

235

236

237

238

239

240

241

242

243

244

245

246

247

248

249

250

251

\section{Expression analysis of DoMYB genes in different tissues}

The transcriptome data of seven tissues including root tips (RT), roots (RO), stems (ST), leaves (LE), flower buds (FB), columns (CO), and sepals (SP) were downloaded from the NCBI SRA (Sequence Read Archives) database. 65 of 101 DoMYB genes were detected in the transcriptome analysis (Fig. 4). They were clustered into five groups (A-E). Among those groups, 17 genes were classified in group A with relatively high expression levels in roots and/or stems. Four genes in group B displayed low expression levels in various tissues. Out of 65 genes, 22 genes were clustered in group C. They had the highest transcript abundance in the roots and/or root tips. All 12 genes in group D exhibited a high expression levels in columns and/or sepals. The remaining ten genes were all in group E. They had higher expression in flower buds if compared with that in other tissues. Moreover, several genes showed tissue-specific expression patterns. For example, seven genes were only expressed in flowers (DoMYB4, DoMYB12, DoMYB40, DoMYB52, DoMYB58, DoMYB86, DoMYB94), three genes were only expressed in roots (DoMYB6, DoMYB78, DoMYB81) (Supplementary Table S3).

D. officinale is a valuable medicinal plant for its high content of secondary metabolites, including polysaccharides, alkaloids and terpenes. Due to its strict requirements on the growth environment, it usually suffers from various biotic or abiotic stresses during its development and growth. Based on the reported functions of $R 2 R 3-M Y B$ in other plants, we screened out 20 DoMYBs belonged to six subfamilies to study the role of $R 2 R 3-M Y B$ in the regulation of secondary metabolism and the responses to abiotic stress for D. officinale (Stracke et al., 2007; Adato et al., 2009). Their expression patterns in different tissues (Fig. 5) and under various abiotic stresses were tested (Fig. 6, 7). Among the five genes of the S1 subfamily, DoMYB30, DoMYB49, and DoMYB101 showed higher expression levels in flowers, DoMYB29 had higher expression levels in protocorms, and DoMYB93 displayed higher expression levels in stems. Otherwise, five genes of the S4 subfamily showed higher expression levels in flowers. Among the S7 subfamily, DoMYB26 had the highest expression level in stems, and DoMYB32 had the 
252 highest expression level in flowers. Among the S9 subfamily, DoMYB11 and DoMYB46 had

253 higher expression levels in flowers, and DoMYB31 had higher expression level in stems. In the 254 S19 subfamily, DoMYB40 and DoMYB52 had higher expression levels in flowers. In the S22 255 subfamily, DoMYB4 and DoMYB8 had higher expression levels in flowers. Otherwise, 256 DoMYB20 had higher expression level in roots. These results are in accord with transcriptome 257 data.

\section{Expression pattern of $D o M Y B$ genes in response to abiotic stress in protocorms}

To explore the functions of the $R 2 R 3-M Y B$ genes in $D$. officinale, the expression patterns of 260 $20 D o M Y B$ genes were analyzed under drought and salt stress. Multiple genes were upregulated,

261 yet a few genes were downregulated in response to drought and salt stress. DoMYB20, DoMYB29

262 and DoMYB69 showed downregulated expression patterns under either drought or salt stress.

263 Five DoMYB genes, DoMYB31, DoMYB40, DoMYB49, DoMYB52 and DoMYB54, showed

264 increased expression levels at different times under the drought treatment (Fig. 6). The 265 expression levels of DoMYB31, DoMYB40 and DoMYB54, were significantly increased after one 266 day of treatment. The expression of DoMYB49 increased after seven days of treatment. 267 DoMYB52 exhibited an increased expression level after three days of treatment. Also, 268 DoMYB26, DoMYB31 and DoMYB67 were identified to respond to salt stress (Fig. 7). DoMYB26

269 had an increased expression level after one day of treatment. DoMYB31 showed an increased 270 expression level with approximately 40-fold after four days of treatment. DoMYB67 exhibited an 271 elevated expression level after seven days of treatment.

272 Co-expression network analysis of $D o M Y B$ genes in different tissues and different 273 treatments

274

To analyze the relationship of $20 D o M Y B$ genes, a co-expression network was constructed 275 using Cytoscape 3.3. Based on the data of the relative transcript abundance in different tissues under various kinds of treatments, a tissue co-expression network and a post-processing gene coexpression network were constructed to explore the relationship of the $M Y B$ genes in $D$. officinale (Supplementary Fig. S4A/B). The results showed that the tissue co-expression network contained 17 nodes (DoMYB Genes) and 66 edges (co-expressed gene pairs), representing PCCs between tissue co-expressing events. Moreover, the processed co-expression network contained

28116 nodes and 44 edges, representing the handling of PCCs between co-expression events. Each 282 node had a different number of regulatory edges, ranging from 1 to 11. 


\section{Discussion}

284 MYB transcription factors (TFs) are a large gene family in plants. Previous studies have 285 identified many MYB genes in Orchidaceae. For example, by RT-PCR, $21 R 2 R 3-M Y B$ genes 286 have been isolated from the Dendrobium orchid hybrid Woo Leng. Four of these genes, $287 D w M Y B 1, D w M Y B 2, D w M Y B 8$, and $D w M Y B 10$, belong to typical plant $R 2 R 3-M Y B$ genes (Wu 288 et al., 2003). Otherwise, three R2R3-MYB transcription factors, including PeMYB2, PeMYB11 289 and PeMYB12, have been identified and proven to be concomitant with the red color formation 290 in flowers from Phalaenopsis spp (Hsu et al., 2015). Similarly, DhMYB1 has been confirmed to 291 be involved in the development of the conical cell shape of epidermis cells of the Dendrobium 292 hybrid flower labellum (Lau et al., 2015). In this study, 101 DoMYBs and 99 PaMYBs of R2R3$293 M Y B$ genes were identified from genomic database sequences and classified into 22 subfamilies.

294 In contrast to the $R 2 R 3-M Y B$ gene numbers found in other species, the numbers of $R 2 R 3-M Y B$ 295 genes in D. officinale and P. aphrodite were less than those in A. thaliana (126), O. sativa (109), 296 Z. mays (157) and P.trichocarpa (192). All of the identified R2R3-MYB genes were divided into 297 different subfamilies. The subfamilies S14, S18 and S21, contained the most MYBs. Nine 298 subfamilies included the same number of $R 2 R 3-M Y B$ genes from both $D$. officinale and $P$. 299 Aphrodite. There were no R2R3-MYB members found in the S6, S12, and S15 R2R3-MYB 300 subfamilies. These results may imply the special characteristics $R 2 R 3-M Y B$ genes in two 301 Orchidaceae species (Fig. 2).

302 It has been reported that several $R 2 R 3-M Y B$ genes are involved in regulating responses to 303 biotic and abiotic stresses. For example, the S1 subfamily genes AtMYB96 and OsMYB4 were 304 confirmed to be involved in the drought response of plants (Seo et al., 2009; Vannini et al., 305 2004). AtMYB41 and AtMYB102 contribute to plant resistance against wounding and osmotic 306 stress (Denekamp et al., 2003; Lippold et al., 2009). Overexpression of AtMYB2 can be induced

307 by dehydration and salt stress (Abe et al., 2003). AtMYB62 participates in response to phosphate 308 starvation (Devaiah et al., 2009). Besides, the S22 subfamily gene AtMYB44 has been shown to 309 be induced by drought and high salt. Overexpression of AtMYB44 obviously enhances drought 310 and salt tolerance in plants (Jung et al., 2007). AmMYB1 is related to the enhancement of 311 tolerance to $\mathrm{NaCl}$ stress in transgenic tobacco (Ganesan et al., 2012). In this study, 20 R2R3-

$312 M Y B$ genes were tested in response to drought and salt stress treatments. Significant changes in 313 the expression levels of all of the members of the S19 subfamily were detected (Fig. 6 and Fig. 314 7). For example, DoMYB40 showed increased expression after one day of drought treatment. 
315 Then, the expression level of DoMYB40 gradually decreased. DoMYB52 displayed the highest

316 expression level after three days of treatment. On the other hand, after undergoing $\mathrm{NaCl}-$

317 simulated salt stress, DoMYB40 reached the highest expression level under treatment for three

318 days, after which, its expression level decreased. DoMYB52 expression increased after four days

319 of treatment and decreased after that. These results may imply the vital role of the S19 subfamily

320 members in response to abiotic stress.

\section{Conclusions}

322 In this study, 101 and $99 R 2 R 3-M Y B$ genes were identified from the genomes of Dendrobium 323 officinale and Phalaenopsis aphrodite. They were distributed into 22 subfamilies based on the 324 homologous analysis. To test the function of identified $R 2 R 3-M Y B$ genes, the expression pattern 325 analysis of the DoMYB genes from six subfamily members in different tissues revealed that the 326 S4 and S19 subfamily members had the highest gene expression levels in flowers. Besides, 327 determination of expression level of $D o M Y B s$ verified that some of them were responsible for the 328 stress response. These results may provide useful information for further studies of the R2R3329 MYB gene family.

\section{Acknowledgments}

331 This work was supported by a scholarship from the China Scholar Council and Anhui Provincial 332 Department of Education (gxfx2017006), Science and Technology Major Project in Anhui 333 province (17030701031) and Anhui Natural Science Foundation (1908085MC59).

\section{Author Contributions}

335 H.H. Fan and M.L. Cui designed the experiment, performed the experiment procedure and wrote 336 the manuscript. N.H. Li performed gene expression experiment, analyzed data. X.J. Li helped 337 with the experiment. Y.X. Liang helped a lot in the process of revision. L. Liu helped revise the 338 manuscript. Y.P. Cai helped with data analysis. Y. Lin designed the experiment, supervised the 339 research and revised the manuscript before submitting it to the journal.

\section{Additional Information}

341 Supplementary Materials: Supplementary Table S1: The correspondence between gene serial 342 number and nomenclature, Supplementary Table $\mathrm{S} 2: \mathrm{Ka} / \mathrm{Ks}$ analysis of $R 2 R 3-M Y B$ genes from $D$. 343 officinale and $P$. aphrodite, Supplementary Table S3: The FPKM values of R2R3-MYB genes in 
344 different tissues of $D$. officinale, Supplementary Table S4: Primer sequences of partial subfamily 345 members of DoMYB genes, Supplementary Table S5: Blast2Go annotation details of MYB 346 protein sequences, Supplementary Table S6: The similarity values of $P a M Y B$ cluster, 347 Supplementary Fig. S1: Phylogenetic relationships, intron pattern, and architecture of conserved 348 protein motifs in R2R3-MYB proteins from $P$. aphrodite, Supplementary Fig. S2: Gene 349 Ontology (GO) analysis of $R 2 R 3-M Y B$ genes from D. officinale (A/B) and P. aphrodite (C/D),

350 Supplementary Fig. S3: Chromosomal location analysis of the PaMYB genes, Supplementary Fig. 351 S4: Co-expression network of DoMYB genes in tissues (A) and their responses to salt, drought, 352 MeJA, SA, and ABA treatments (B).

353 Conflicts: The authors declare no conflict of interest.

354

355

\section{References}

356

Adato A, Mandel T, Mintz-Oron S, Venger I, Levy D, Yativ M, Domínguez E, Wang Z, De Vos RC, Jetter R, Schreiber L, Heredia A, Rogachev I, Aharoni A. 2009. Fruit-Surface Flavonoid Accumulation in Tomato Is Controlled by a SIMYB12-Regulated Transcriptional Network. PLOS Genetics 5, e1000777.

Abe H, Urao T, Ito T, Seki M, Shinozaki K, Yamaguchi-shinozaki K. 2003. Arabidopsis AtMYC2 (bHLH) and AtMYB2 (MYB) function as transcriptional activators in abscisic acid signaling. Plant Cell 15, 63-78.

Bailey TL, Williams N, Misleh C, Li WW. 2006. MEME: discovering and analyzing DNA and protein sequence motifs. Nucleic Acids Research 34(Web Server issue), 369-373.

Cao YP, Han YH, Li DH, Lin Y, Cai YP. 2016. MYB Transcription Factors in Chinese Pear (Pyrus bretschneideri Rehd.): Genome-Wide Identification, Classification, and Expression Profiling during Fruit Development. Frontiers in Plant Science 7, 1-14.

Chao YT, Chen WC, Chen CY, Ho HY, Yeh CH, Kuo YT, Su CL, Yen SH, Hsueh HY, Yeh JH, Hsu HL, Tsai YH, Kuo TY, Chang SB, Chen KY, Shih MC. 2018. Chromosome-level assembly, genetic and physical mapping of Phalaenopsis aphrodite genome provides new insights into species adaptation and resources for orchid breeding. Plant Biotechnology Journal 16, 2027-2041.

Chen BJ, Wang Y, Hu YL, Wu Q, Lin ZP. 2005. Cloning and characterization of a droughtinducible MYB gene from Boea crassifolia. Plant Science 168, 493-500. 
375 Conesa A, Gotz S, Garcia-Gomez JM, Terol J, Talon M, Robles M. 2005. Blast2GO: a universal 376 tool for annotation, visualization and analysis in functional genomics research. 377 Bioinformatics 21, 3674-3676.

378 Denekamp M, Smeekens SC. 2003. Integration of wounding and osmotic stress signals 379 determines the expression of the AtMYB102 transcription factor gene. Plant Physiology $380 \quad 132,1415-1423$.

381 Devaiah BN, Madhuvanthi R, Karthikeyan AS, Raghothama KG. 2009. Phosphate starvation 382 383 responses and gibberellic acid biosynthesis are regulated by the MYB62 transcription factor in Arabidopsis. Molecular Plant 2, 43-58.

384 Du H, Feng BR, Yang SS, Huang YB, Tang YX. 2012. The R2R3-MYB Transcription Factor 385 Gene Family in Maize. PLOS ONE 7, e37463.

Dubos C, Strack R, Grotewold E, Weisshaar B, Martin C, Lepiniec L. 2010. MYB transcription factors in Arabidopsis. Trends in Plant Science 15, 573-581.

Ganesan G, Sankararamasubramanian HM, Harikrishnan M, Ashwin G, Parida A. 2012. A MYB transcription factor from the grey mangrove is induced by stress and confers $\mathrm{NaCl}$ tolerance in tobacco. Journal of Experimental Botany 63, 4549-4561.

Guo AY, Zhu QH, Chen X, Luo JC. 2007. GSDS: a gene structure display server. Hereditas (Beijing) 29(8), 1023-1026.

Hsu CC, Chen YY, Tsai WC, Chen WH, Chen HH. 2015. Three R2R3-MYB transcription factors regulate distinct floral pigmentation patterning in Phalaenopsis orchids. Plant Physiology 168, 175-191.

Jin Q, Yao Y, Cai YP, Lin Y. 2013. Molecular cloning and sequence analysis of a phenylalanine ammonia-lyase gene from Dendrobium. PLOS ONE 8, e62352.

Jung C, Seo JS, Han SW, Koo YJ, Kim CH, Song SI, Nahm BH, Choi YD, Cheong JJ. 2007. Overexpression of AtMYB44 enhances stomatal closure to confer abiotic stress tolerance in transgenic Arabidopsis. Plant Physiology 146, 623-635.

Katiyar A, Smita S, Lenka SK, Rajwanshi R, Chinnusamy V, Bansal KC. 2012. Genome-wide classification and expression analysis of MYB transcription factor families in rice and Arabidopsis. BMC Genommics 13, 544-562.

404 Kim D, Pertea G, Trapnell C, Pimentel H, Kelley R, Salzberg SL. 2013. TopHat2: Accurate 405 alignment of transcriptomes in the presence of insertions, deletions and gene fusions. Genome Biology 14, R36. 
407 Kirik V, Kolle K, Wohlfarth T, Misera S, Baumlein H. 1998. Ectopic expression of a novel

408

409

410

411

412

413

414

415

416

417

418

419

420

421

422

423

424

425

426

427

428

429

430

431

432

433

434

435

436

437

MYB gene modifies the architecture of the Arabidopsis inflorescence. The Plant Journal 13, 729-742.

Lau SE, Schwarzacher T, Othman RY, Harikrishna JA. 2015. dsRNA silencing of an R2R3-MYB transcription factor affects flower cell shape in a Dendrobium hybrid. BMC Plant Biology $15,194$.

Li R, Reddy VA, Jin J, Rajan C, Wang Q, Yue G, Lim CH, Chua NH, Ye J, Sarojam R. 2017. Comparative transcriptome analysis of oil palm flowers reveals an EAR-motif containing R2R3-MYB that modulates phenylpropene biosynthesis. BMC Plant Biology 17, 219.

Li WB, Ding ZH, Ruan MB, Yu XL, Peng M, Liu YF. 2017. Kiwifruit R2R3-MYB transcription factors and contribution of the novel $A c M Y B 75$ to red kiwifruit anthocyanin biosynthesis. Scientific Reports 7, 16861.

Librado P, Rozas J. 2009. DnaSP v5: A software for comprehensive analysis of DNA polymorphism data. Bioinformatic 25, 1451-1452.

Lippold F, Sanchez DH, Musialak M, Schlereth A, Scheible WR, Hincha DK, Udvardi, MK. 2009. AtMyb41 regulates transcriptional and metabolic responses to osmotic stress in Arabidopsis. Plant Physiology 149, 1761-1772.

Livak KJ, Schmittgen TD. 2001. Analysis of relative gene expression data using real-time quantitative PCR and the 2(-Delta Delta C(T)) Method. Methods 25, 402-408.

Matus JT, Aquea F, Arce-Johnson P. 2009. Analysis of the grape MYB R2R3 subfamily reveals expanded wine quality-related clades and conserved gene structure organization across Vitis and Arabidopsis genomes. BMC Plant Biology 8, 83.

Nesi N, Jond C, Debeaujon I, Caboche M, Lepiniec L. 2001.The Arabidopsis TT2 gene encodes an R2R3 MYB domain protein that acts as a key determinant for proanthocyanidin accumulation in developing seed. Plant Cell 13, 2099-2114.

Peng X, Liu H, Wang D, Shen S. 2016. Genome-wide identification of the Jatropha curcas MYB family and functional analysis of the abiotic stress responsive gene $J c M Y B 2$. BMC Genomics 17(1), 251-251.

Rabinowicz PD, Braun EL, Wolfe AD, Bowen B, Grotewold E. 1999. Maize R2R3-MYB genes: sequence analysis reveals amplification in the higher plants. Genetics 153, 427-444. 
438 Reddy VA, Wang Q, Dhar N, Kumar N, Sarojam R. 2017. Spearmint R2R3-MYB transcription factor $M S M Y B$ negatively regulates monoterpene production and suppresses the expression of geranyl diphosphate synthase large subunit (MsGPPS.LSU). Plant Biotechnology Journal 15, 1105-1119.

Riechmann JL, Heard J, Martin G, Reuber L, Jiang CZ, Keddie J, Adam L, Pineda O, Ratcliffe OJ, Samaha RR, Creelman R, Pilgrim M, Broun P, Zhang JZ, Ghandehari D, Sherman BK, Yu GL. 2000. Arabidopsis transcription factors: Genome-wide comparative analysis among eukaryotes. Science 290, 2105-2110.

Seo PJ, Xiang F, Qiao M, Park JY, Park CM. 2009. The MYB96 transcription factor mediates abscisic acid signaling during drought stress response in Arabidopsis. Plant Physiology $151,275-289$.

Stracke R, Ishihara H, Barsch GHA, Mehrtens F, Niehaus K, Weisshaar B. 2007. Differential regulation of closely related $R 2 R 3-M Y B$ transcription factors controls flavonol accumulation in differene parts of Arabidopsis thaliana seeding. The Plant Journal 50, 660-677.

Stracke R, Werber M, Weisshaar B. 2001.The R2R3-MYB gene family in Arabidopsis thaliana. Current Opinion in Plant Biology 4, 447-456.

Tamura K, Peterson D, Peterson N, Stecher G, Nei M, Kumar S. 2011. MEGA5: molecular evolutionary genetics analysis using maximum likelihood, evolutionary distance, and maximum parsimony methods. Molecular Biology and Evolution 28, 2731-2739.

Tang J, Wang F, Wang Z, Huang ZN, Xiong AS, Hou XL. 2013. Characterization and coexpression analysis of WRKY orthologs involved in responses to multiple abiotic stresses in Pak-choi (Brassica campestris ssp. chinensis). BMC Plant Biology 13, 188.

Trapnell C, Roberts A, Goff L, Pertea G, Kim D, Kelley DR, Pimentel H, Salzberg SL, Rinn JL, Pachter L. 2012. Differential gene and transcript expression analysis of RNA-seq experiments with Tophat and Cufflinks. Nature Protocols 7, 562-578.

Vannini C, Locatelli F, Bracale M, Magnani E, Marsoni M, Osnato M. 2004. Overexpression of the rice $O s m y b 4$ gene increases chilling and freezing tolerance of Arabidopsis thaliana plants. Plant Journal 37, 115-127.

Wilkins O, Nahal H, Foong J, Provart NJ, Campbell MM. 2009. Expansion and Diversification of populus R2R3-MYB Family of Transcription Factors. Plant Physiology 149, 981-993. 
$469 \mathrm{Wu}$ XM, Lim SH, Yang WC. 2003. Characterization, expression and phylogenetic study of $470 \quad$ R2R3-MYB genes in orchid. Plant Molecular Biology 51, 959-972.

471 Ye J. 2006. WEGO: a web tool for plotting GO annotations. Nucleic Acids Research 34 (Web $472 \quad$ Server issue), W293-W297.

473 Zhang GQ, Liu KW, Li Z, Lohaus R, Hsiao YY, Niu SC, Wang JY, Lin YC, Xu Q, Chen 474 LJ, Yoshida K, Fujiwara S, Wang ZW, Zhang YQ, Mitsuda N, Wang M, Liu GH, Pecoraro 475 L, Huang HX, Xiao XJ, Lin M, Wu XY, Wu WL, Chen YY, Chang SB, Sakamoto 476 S, Ohme-Takagi M, Yagi M, Zeng SJ, Shen CY, Yeh CM, Luo YB, Tsai WC, Van de Peer 477 Y, Liu ZJ. 2017. The Apostasia genome and the evolution of orchids. Nature 549, 379-383. 478 Zhang Z, Li J, Zhao XQ, Wang J, Wong GKS, Yu J. 2006. KaKs_Calculator: calculating Ka and 479 Ks through model selection and model averaging. Genomics Proteomics and 480 Bioinformatics 4, 259-263.

481 Zhong RQ, Lee CH, Zhou JL, McCarthy RL, Ye ZH. 2008. A Battery of Transcription Factors 482 Involved in the Regulation of Secondary Cell Wall Biosynthesis in Arabidopsis. Plant Cell 20, 2763-2782. 
Figure 1

Phylogenetic analysis of R2R3-MYB proteins from Dendrobium officinale and Phalaenopsis aphrodite .

Totally, 101 R2R3-MYB proteins from $D$. officinale, 99 R2R3-MYB proteins from $P$. aphrodite and 126 R2R3-MYB proteins from $A$. thaliana were selected to construct the neighbor-joining tree with 1000 bootstraps. S1-S25 indicated the divided subfamilies according to the categories of R2R3-MYB proteins in A. thaliana. 


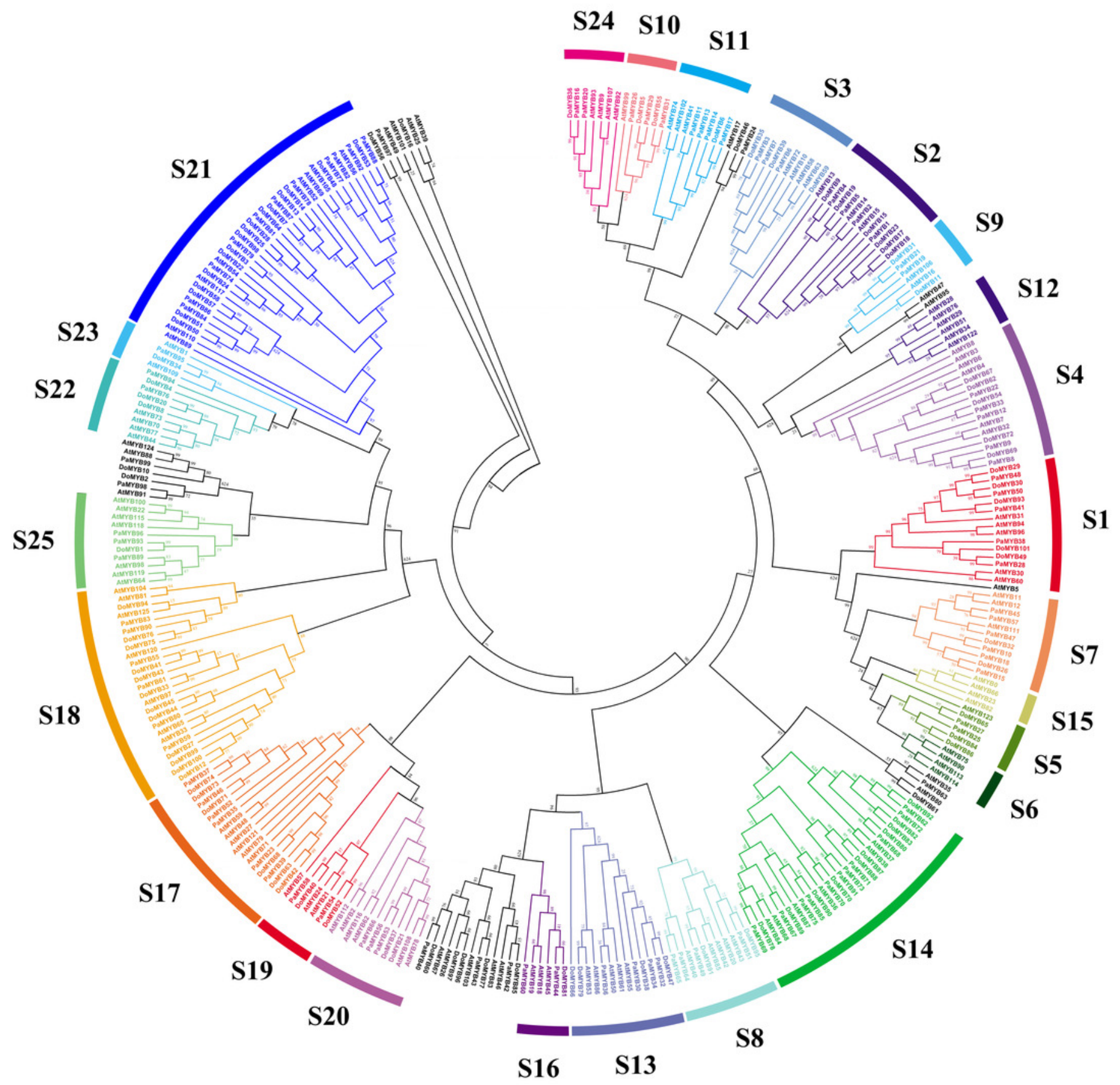




\section{Figure 2}

Phylogenetic relationships, intron pattern, and architecture of conserved protein motifs in R2R3-MYB proteins from $D$. officinale.

A, The phylogenetic tree constructed with 101 R2R3-MYB proteins from D.officinale. S1-S25 indicated the divided subfamilies. B, Architecture of conserved protein motifs in different subfamilies. The colored boxes indicated the different motifs as listed at the bottom of the figure. C, The predicted exon-intron structures. The yellow boxes and black lines exhibited exons and introns, respectively. 


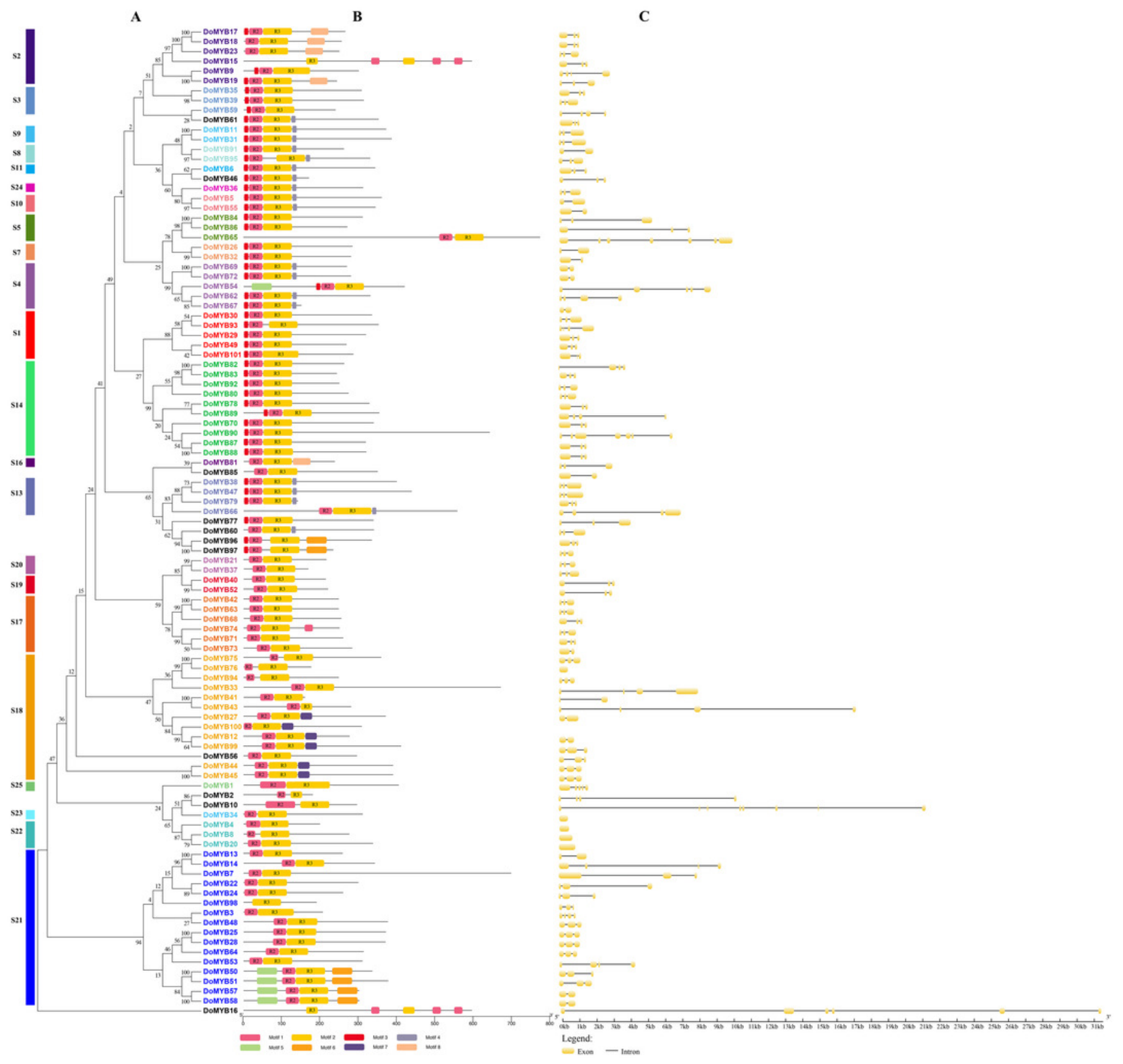


Figure 3

Sequence logos of the R2 $(A \& C)$ and $R 3(B \& D)$ MYB repeats

$A \& B$ respectively indicated the $R 2$ and $R 3$ logos based on multiple alignment analysis of 101 DoR2R3-MYB proteins. C \& D indicated the R2 and R3 logos of 99 PaR2R3-MYB proteins. * indicated typical conserved Trp residues in the MYB domain.
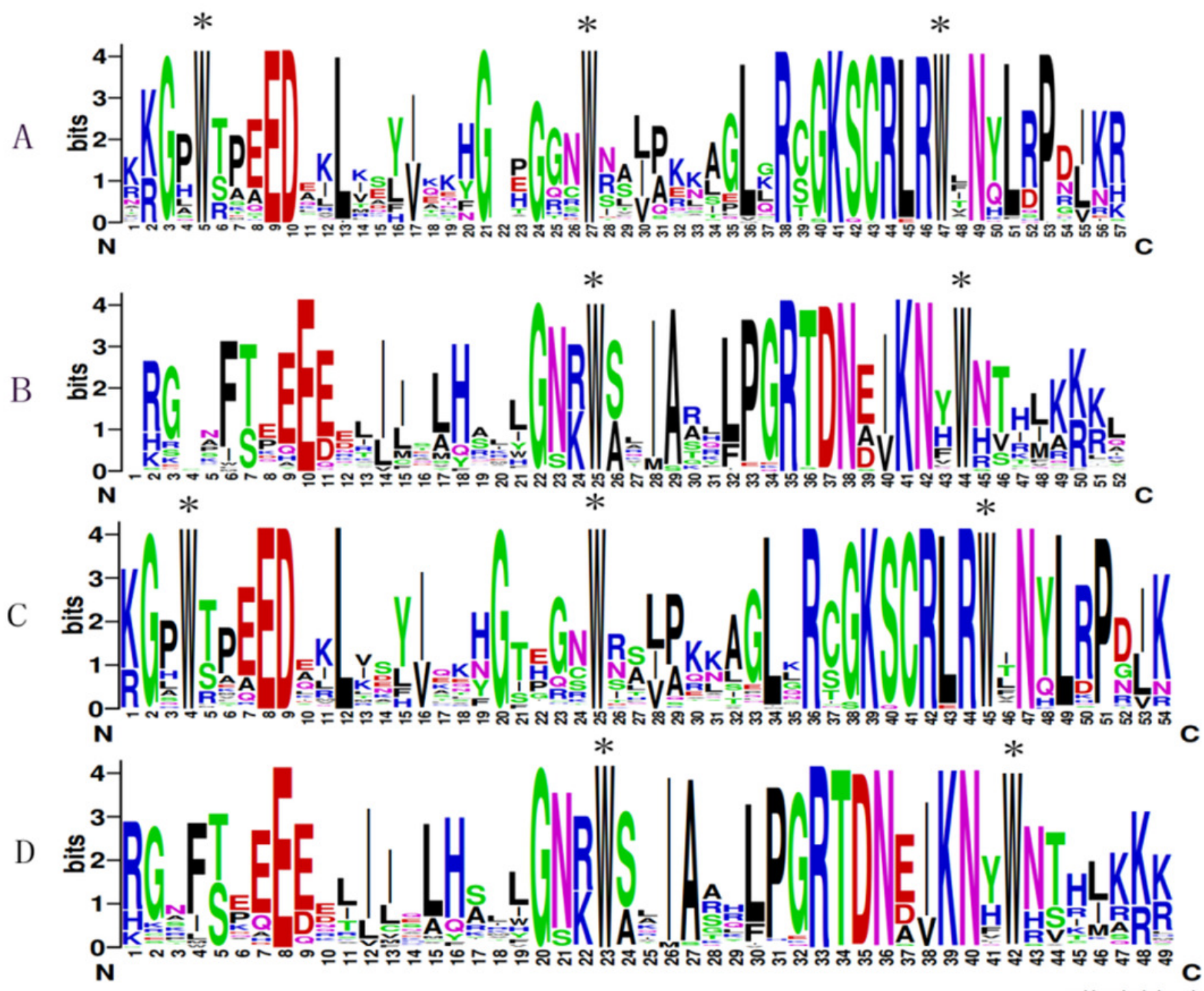


\section{Figure 4}

RNA-seq analysis of R2R3-MYB transcription factors in D. officinale.

The heatmap was generated using $\mathrm{R}$ gplots package, and the FPKM values of $D$. officinale genes were evaluated and normalized based RNA-seq data from NCBI SRA database.

Differential expression pattern of 65 annotated R2R3-MYBs in various tissues, including root tips (RT), roots (RO), stems (ST), leaves (LE), flower buds (FB), columns (CO), and sepals (SP). The expression values are available in Supplementary Table S3. 


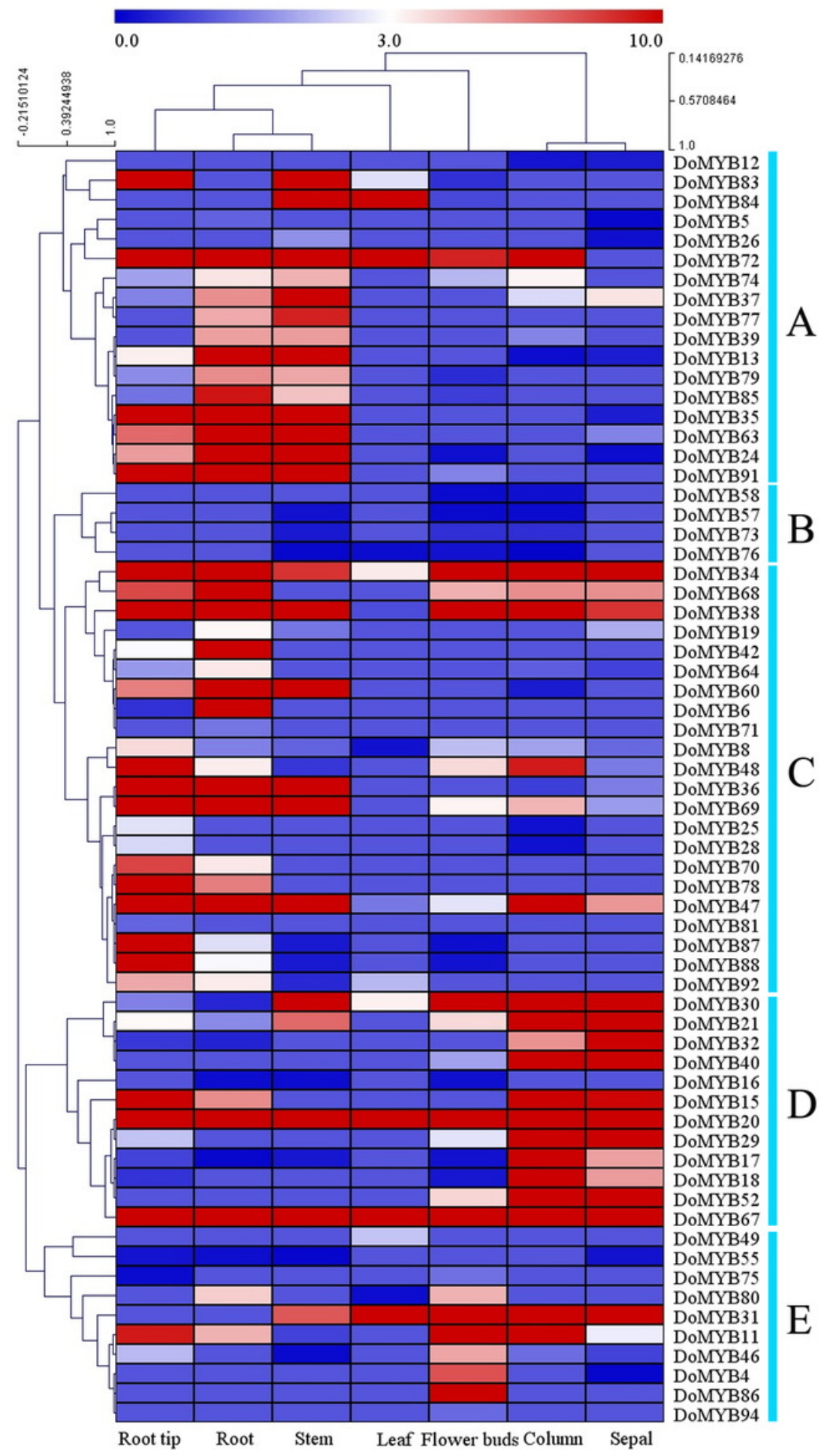




\section{Figure 5}

qRT-PCR validation of R2R3-MYB transcription factors of $D$. officinale in different tissues.

Twenty genes were selected to perform qRT-PCR analysis to verify the transcriptome data.

Three biological repeats were conducted. The average data was used to construct heatmap

by MeV 4.9 with Pearson correlation using average linkage clustering. 


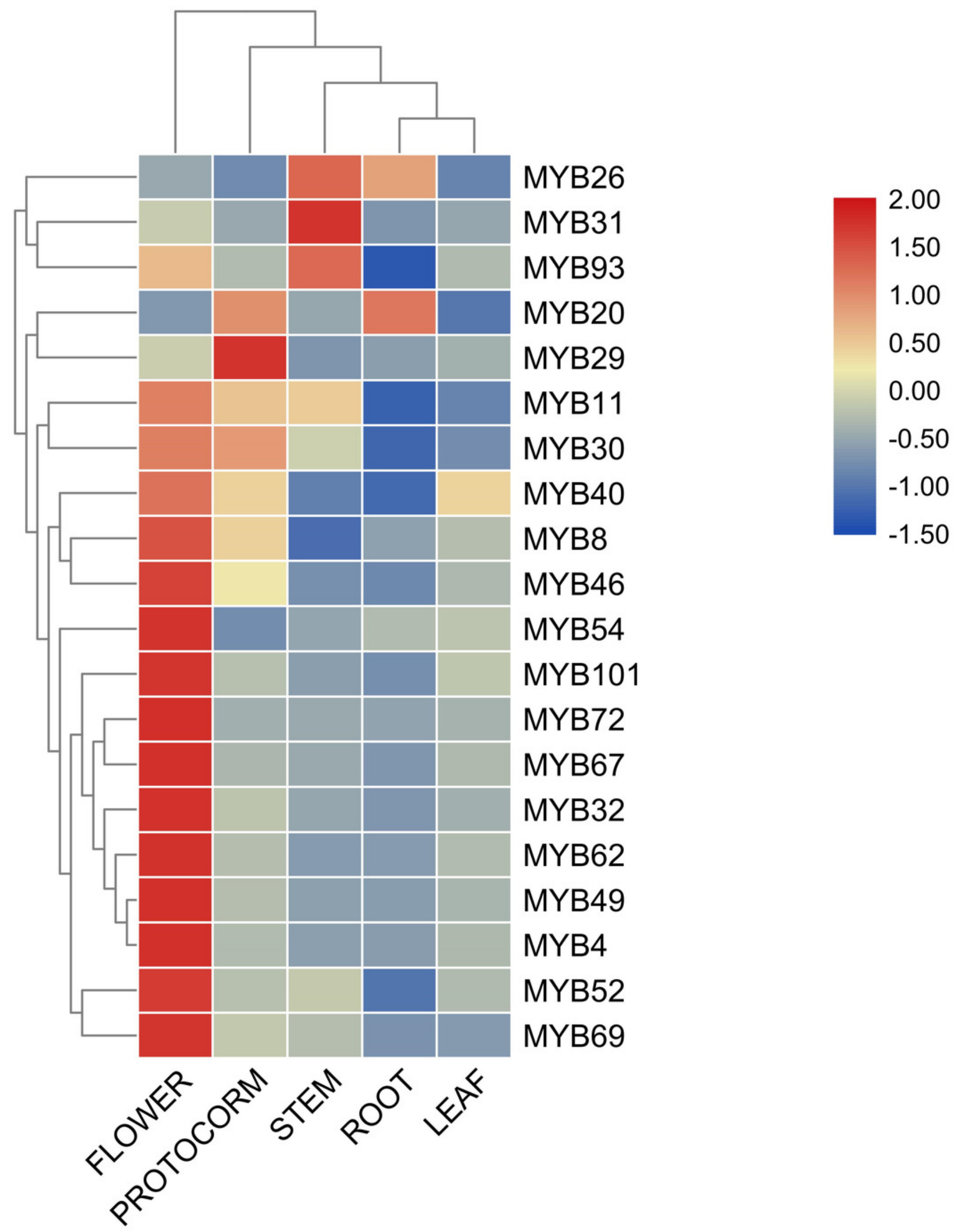




\section{Figure 6}

Expression patterns of the DoMYB genes in response to drought treatment.

The relative expression levels of 20 DoMYB genes were examined by qRT-PCR and normalized to the reference gene $\beta$-actin. Every experiment had three biological repeats. The $x$-axis indicated the time course of each stress treatment, and the $y$-axis represented the relative expression level. Mean values and standard deviations (SDs) indicated by error bars. ** significant difference $(P<0.01)$, *significant difference at $P<0.05$. The details of the letters A-T were listed as follows. A: DoMYB4, B: DoMYB8, C: DoMYB11, D: DoMYB20, E: DoMYB26, F: DoMYB29, G: DoMYB30, H: DoMYB31, I: DoMYB32, J: DoMYB40, K: DoMYB46, L: DoMYB49, M: DoMYB52, N: DoMYB54, O: DoMYB62, P: DoMYB67, Q: DoMYB69, I: DoMYB72, S: DoMYB93, T: DoMYB101. The letters A-T in the following figure are the same. 

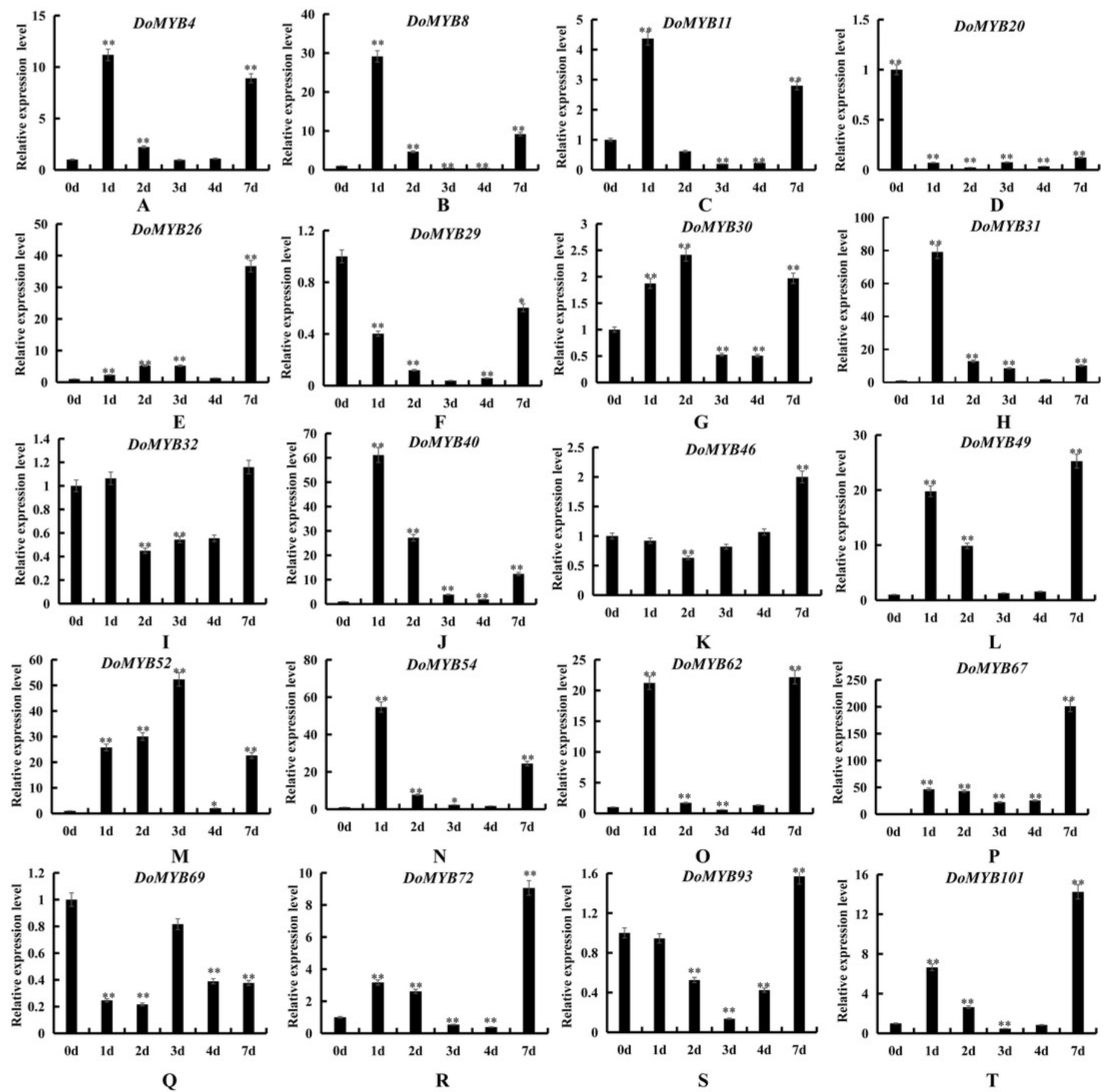


\section{Figure 7}

Expression patterns of the DOMYB genes under salt treatment.

The relative expression level of 20 DoMYB genes was evaluated by qRT-PCR. The gene $\beta$ actin was selected as a reference. Every experiment had three biological repeats. The $\mathrm{x}$-axis indicated the time course of each stress treatment, and the $y$-axis represented the relative expression level. Mean values and standard deviations (SDs) indicated by error bars. ** significant difference $(P<0.01)$, * significant difference at $P<0.05$. The details of the letters A-T were listed as follows. A: DoMYB4, B: DoMYB8, C: DoMYB11, D: DoMYB20, E: DoMYB26, F: DoMYB29, G: DoMYB30, H: DoMYB31, I: DoMYB32, J: DoMYB40, K: DoMYB46, L: DoMYB49, M: DoMYB52, N: DoMYB54, O: DoMYB62, P: DoMYB67, Q: DoMYB69, I: DoMYB72, S: DoMYB93, T: DOMYB101. 

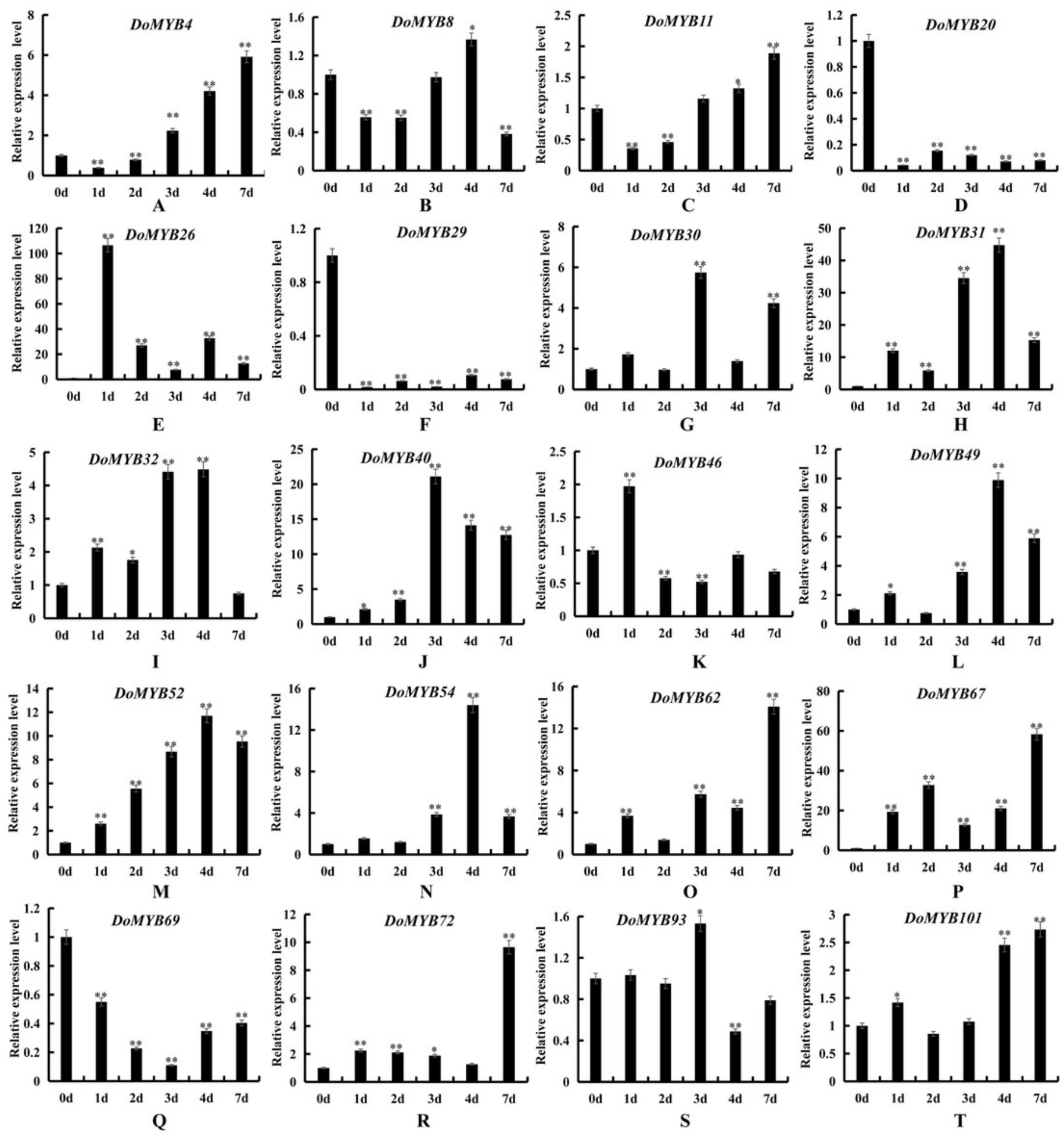